\title{
Bloodstream and endovascular infections due to Abiotrophia defectiva and Granulicatella species
} Laurence Senn¹, José M Entenza1, Gilbert Greub², Katia Jaton², Aline Wenger ${ }^{2}$, Jacques Bille ${ }^{2}$, Thierry Calandra ${ }^{1}$ and Guy Prod'hom*2

\author{
Address: 'Infectious Diseases Service, University Hospital, Lausanne, Switzerland and ${ }^{2}$ Institute of Microbiology, University Hospital, Bugnon 46, \\ 1011 Lausanne CHUV, Switzerland \\ Email: Laurence Senn - laurence.senn@chuv.ch; José M Entenza - jose.entenza@unil.ch; Gilbert Greub - gilbert.greub@chuv.ch; \\ Katia Jaton - Katia.Jaton-Ogay@chuv.ch; Aline Wenger - aline.wenger@chuv.ch; Jacques Bille - jacques.bille@chuv.ch; \\ Thierry Calandra - thierry.calandra@chuv.ch; Guy Prod'hom* - guy.prodhom@chuv.ch \\ * Corresponding author
}

Published: 20 January 2006

BMC Infectious Diseases 2006, 6:9 doi:10.1/86/147/-2334-6-9

This article is available from: http://www.biomedcentral.com/I47I-2334/6/9

(c) 2006 Senn et al; licensee BioMed Central Ltd.

This is an Open Access article distributed under the terms of the Creative Commons Attribution License (http://creativecommons.org/licenses/by/2.0), which permits unrestricted use, distribution, and reproduction in any medium, provided the original work is properly cited.
Received: 06 September 2005

Accepted: 20 January 2006

\begin{abstract}
Background: Abiotrophia and Granulicatella species, previously referred to as nutritionally variant streptococci (NVS), are significant causative agents of endocarditis and bacteraemia. In this study, we reviewed the clinical manifestations of infections due to A. defectiva and Granulicatella species that occurred at our institution between 1998 and 2004.
\end{abstract}

Methods: The analysis included all strains of NVS that were isolated from blood cultures or vascular graft specimens. All strains were identified by I6S rRNA sequence analysis. Patients' medical charts were reviewed for each case of infection.

Results: Eleven strains of NVS were isolated during the 6-year period. Identification of the strains by I6S rRNA showed 2 genogroups: Abiotrophia defectiva (3) and Granulicatella adiacens (6) or "paraadiacens" (2). The three $A$. defectiva strains were isolated from immunocompetent patients with endovascular infections, whereas 7 of 8 Granulicatella spp. strains were isolated from immunosuppressed patients, mainly febrile neutropenic patients. We report the first case of "G. para-adiacens" bacteraemia in the setting of febrile neutropenia.

Conclusion: We propose that Granulicatella spp. be considered as a possible agent of bacteraemia in neutropenic patients.

\section{Background}

Nutritionally variant streptococci (NVS), first described in 1961 by Frenkel and Hirsch [1], were classified on the basis of growth characteristics such as nutrient requirements (pyridoxal) and presence of satellitism. In 1989, based on DNA-DNA hybridisation, Bouvet et al. showed that NVS could be divided in two groups, Streptococcus defectivus and Streptococcus adiacens [2]. In 1995, based on the genetic and phylogenetic analysis of the 16S rRNA sequences, the genus Abiotrophia and the two species $A$. defectiva and A. adiacens were proposed by Kawamura [3]. In 1998, Roggenkamp et al. proposed the new species $A$. elegans [4], and in 1999, Lawson et al. proposed the species A. balaenopterae [5]. Based on 16S rRNA heterogeneity and phenotypic differences, Kanamoto et al. proposed an additional species "Abiotrophia para-adiacens" [6]. In 2000, 


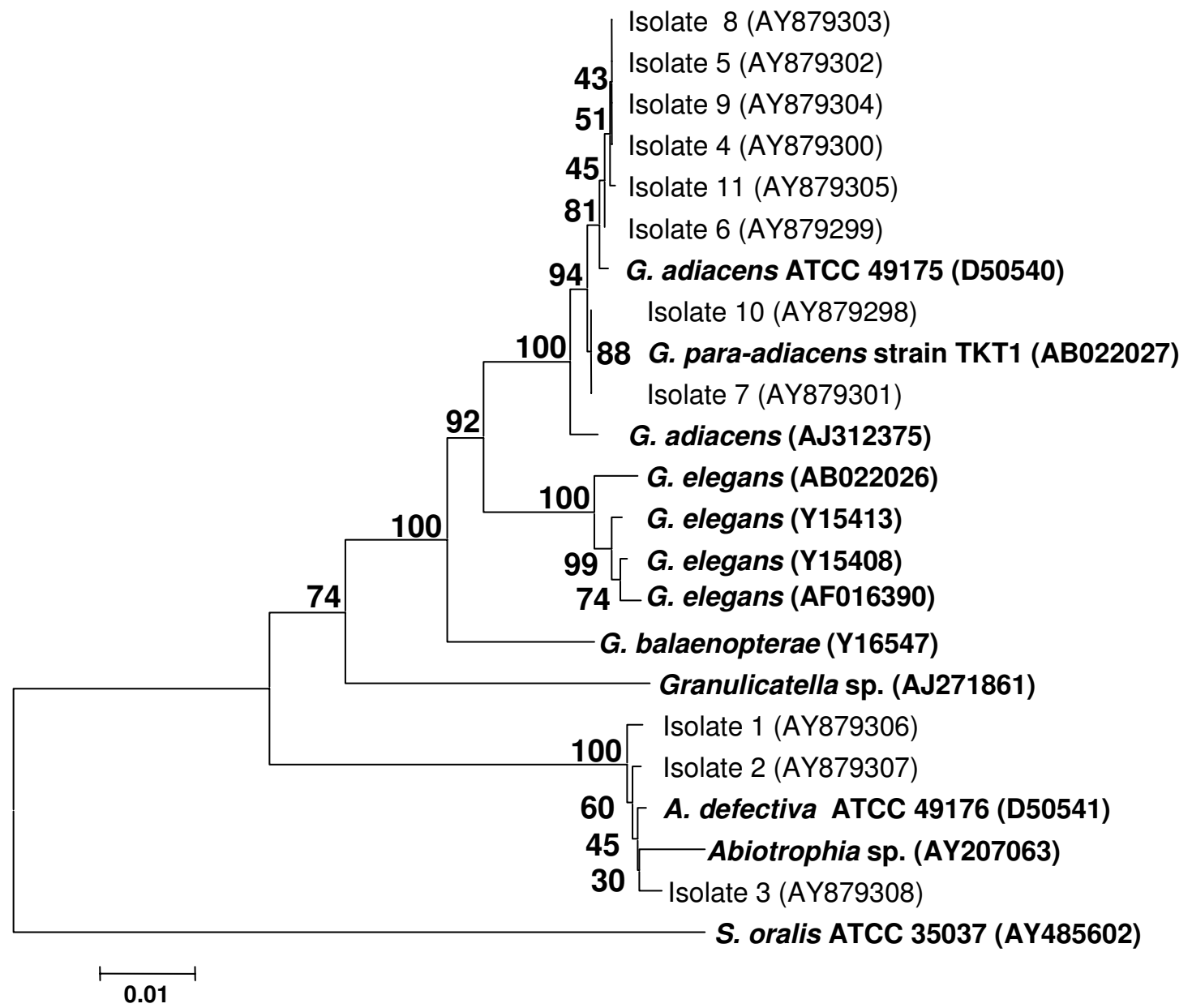

\section{Figure I}

Phylogenetic analysis of the strains. Phylogenetic tree showing the affiliation of 3 isolates to A. defectiva, 6 isolates to $G$. adiacens and 2 isolates to "G. para-adiacens". The tree was inferred from I3I 5 base pairs I6S rRNA sequence data by the neighbour-joining method using the Kimura-corrected p-distance. Streptococcus oralis was used as outgroup. Genbank accession numbers are shown in parentheses.

A. adiacens, A. balaenopterae and A. elegans were reclassified in the new genus Granulicatella by Collins and Lawson [7].

Abiotrophia and Granulicatella species form part of the normal flora of the oral cavity [8-10], the genitourinary tract, and the intestinal tract [11]. G. adiacens is isolated more frequently from oral specimens than other NVS $[4,9,10]$. Bacteraemia and endocarditis are the more frequently reported clinical infections due to Abiotrophia and Granulicatella species [12] and account for 4.3 to $6 \%$ of all "streptococcal" endocarditis [13]. Isolated cases of keratitis [14], endophthalmitis [15], central nervous system infections [16-20], sinusitis, otitis media, prostatitis, cholangitis, arthritis [21-23] and osteomyelitis [24,25] have also been reported. The high prevalence of betalactam and macrolide resistance among isolates of Abio- trophia and Granulicatella may pose a challenge to treat invasive infections [26-28].

In this study, we reviewed the clinical manifestations of infections due to A. defectiva and Granulicatella species that occurred at our institution over a 6 -year period.

\section{Methods}

\section{Bacterial strains}

The analysis included all strains of NVS that were isolated from blood cultures or vascular graft specimens from patients admitted to our 800-bed University Hospital from January 1998 to December 2004. The automated blood culture system used in the microbiology laboratory during the study period was the Bactec 9240 (Becton Dickinson, Sparks, Md.) with the Plus aerobic/F and Lytic 
anaerobic/F vials (Becton Dickinson). The strains were identified to the species level using the Rapid ID32 STREPT system (Bio Mérieux SA, Marcy-l'Etoile, France).

\section{I6S rRNA gene sequencing}

All strains were also identified by $16 \mathrm{~S}$ rRNA sequence analysis. DNA was extracted with the MagNA Pure LC DNA isolation Kit I (Roche Diagnostics, Mannheim, Germany) according to the instructions of the manufacturer. Polymerase chain reaction (PCR) amplification of the $16 \mathrm{~S}$ RNA gene was performed with primers fD1 and rP2 [29] and Taq DNA polymerase (Gibco BRL, Life Technologies) followed by electrophoresis of the PCR products on ethidium bromide-stained 1\% agarose gel. PCR products were purified using the QIAquick PCR purification kit (Qiagen, Courtaboeuf, France). Sequencing was performed by using the dRhodamine Terminator Cycle Sequencing Ready Reaction kit with one of six different primers and AmpliTaq DNA (Perkin-Elmer Biosystems, Warrington, England) with a 3100 ABI Prism automated sequencer (Applied Biosystems, Courtaboeuf, France). Sequences derived from each primer were aligned and combined into a single $16 \mathrm{~S}$ rRNA sequence by using Contig Express, a component of the Vector NTI suite 9.0 (Informax, Frederick, MD). Each sequence was compared with all eubacterial 16S rRNA sequences available in the GenBank database by using the BLASTN 2.2.2 program available on the National Center for Biotechnology Information website [30,31]. The 16S rRNA sequences of Abiotrophia and Granulicatella isolates were aligned with those of other members of the genus Abiotrophia and Granulicatella by using the CLUSTWAL W program supported by the DDBJ website [32]. Sequences were edited by removal of the longer 5' and 3 ' ends so that their lengths matched that of the shortest sequence and then analysed by neighbourjoining, parsimony and minimum evolution methods (Kimura's correction, pairwise deletion option) using the Mega 2.1 software [33]. GenBank accession numbers are shown in Figure 1.

\section{Antimicrobial susceptibility testing}

The minimal inhibitory concentrations (MIC) of penicillin, ceftriaxone, meropenem, clarithromycin, erythromycin, quinupristin/dalfopristin, levofloxacin, vancomycin and teicoplanin were determined for each isolate by the Etest method (AB Biodisk, Solna, Sweden), using Brucella agar supplemented with haemin, vitamin $\mathrm{K} 1$, cystein and $5 \%$ sheep blood as test medium (BA). For vancomycin, an E-test was also performed on Mueller-Hinton agar with $5 \%$ sheep blood (BMH) and 1\% vitox defined supplement (Oxoid, Basel, Switzerland). Antimicrobial susceptibilities were interpreted according to the guidelines established by the CLSI for Streptococcus spp. other than Streptococcus pneumoniae [34].

\section{Clinical data}

The patients' medical charts were reviewed and clinical characteristics (age, sex, clinical diagnosis, underlying conditions, predisposing factors, antibiotic treatment and outcome) were recorded for each case of infection due to NVS. Neutropenia was defined as a neutrophil count of $<500$ cells $/ \mathrm{mm}^{3}$.

\section{Ethics}

The design of this study was in accordance with the ethical standards of our hospital ethics committee. Because the study was retrospective, informed consent was not required.

\section{Results}

During the six-year study period, ten patients had positive blood cultures for NVS and one had a positive culture of a vascular graft fragment. All but one positive blood culture were detected within the first twenty-four hours of incubation. The strains grew equally from both vials. All strains showed satellitism around streaks of Staphylococcus aureus. Seven of 11 strains were identified successfully to the species level using the Rapid ID32 STREPT system.

Based on their 16S rRNA sequences, 3 strains were identified as A. defectiva (isolates 1-3) and 8 as Granulicatella spp. (isolates 4-11). Isolates 1, 2 and 3 exhibited 99.6 to $99.7 \% 16 \mathrm{~S}$ rRNA homology with A. defectiva strain ATCC 49176 and 92.1 to $92.4 \%$ 16S rRNA homology with $G$. adiacens strain ATCC 49175. Isolates 4 to 11 exhibited 92.2 to $92.5 \% 16 \mathrm{~S}$ rRNA homology with A. defectiva strain ATCC 49176 and $99.8 \%$ 16S rRNA homology with $G$. adiacens strain ATCC 49175. Two strains, isolates 7 and 10, exhibited 100\% 16S rRNA homology with "G. paraadiacens" strain TKT1 [6]. The phylogenetic tree shown in Fig. 1 demonstrates the affiliation of strains 1,2 and 3 to the genus Abiotrophia. Phylogenetic analyses confirmed affiliation of strains $4,5,6,8,9$ and 11 to G. adiacens and strains 7 and 10 to "G. para-adiacens" (Fig. 1). Strains 7 and 10 clustered with "G. para-adiacens" strain TKT1 with bootstraps of $94 \%, 84 \%$ and $80 \%$ in neighbour-joining, parsimony and minimum evolution analysis, supporting the node separating them from their closest neighbour $G$. adiacens.

According to CLSI interpretive criteria, the 3 A. defectiva strains were susceptible to all the antibiotics tested except 1 strain with a reduced susceptibly to penicillin (MIC 0.25 $\mu \mathrm{g} / \mathrm{ml}$ ). Two Granulicatella spp. showed resistance to penicillin (MIC 4 and $8 \mu \mathrm{g} / \mathrm{ml}$, respectively), and intermediate susceptibility (MIC $2 \mu \mathrm{g} / \mathrm{ml}$ ) or resistance (MIC $8 \mu \mathrm{g} /$ $\mathrm{ml}$ ) to ceftriaxone. One Granulicatella spp. was intermediately susceptible to penicillin (MIC $0.25 \mu \mathrm{g} / \mathrm{ml}$ ) but sensitive to other beta-lactams. Two other strains were intermediately susceptible to ceftriaxone alone (MIC $2 \mu \mathrm{g} /$ 
$\mathrm{ml}$ ). All Granulicatella spp. strains showed vancomycin MICs of 1.5 to $2 \mu \mathrm{g} / \mathrm{ml}$ and teicoplanin MICs of $<0.25 \mu \mathrm{g} /$ $\mathrm{ml}$ with BA. MICs to vancomycin were lower when tested on BMH (MIC 0.5 to $1 \mu \mathrm{g} / \mathrm{ml}$ ). All Granulicatella spp. strains were susceptible to meropenem, clarithromycin, erythromycin, quinupristin/dalfopristin, and levofloxacin.

The epidemiological and clinical characteristics of the patients are shown in Additional file 1. Two patients with A. defectiva infections had endocarditis, one of which also had sacroileitis. The third patient had polymicrobial ( $A$. defectiva plus Escherichia coli) vascular graft infection related to an aorto-enteric fistula.

Seven of 8 patients with bacteraemia due to Granulicatella spp. were immunosuppressed. Underlying conditions included haematological malignancies (5), lung cancer (1) and advanced metastatic oesophageal cancer (1). Three patients had polymicrobial infections (G. adiacens plus Clostridium sordellii, Staphylococcus epidermidis or Lactobacillus rhamnosus). Six patients presented with febrile chemotherapy-induced neutropenia and mucositis, including one patient with possible infection of a catheter and one with possible endocarditis. One of the six patients with neutropenia died 10 days after bacteraemia from gastrointestinal bleeding in the setting of refractory thrombopenia. The other 5 neutropenic patients clinically improved clinically with intravenous antibiotic therapy. The 3 patients with primary bacteraemia due to Granulicatella spp. with intermediate susceptibility or resistance to penicillin were successfully treated (cases 7, 10 and 11).

\section{Discussion}

A. defectiva and Granulicatella spp. are now considered as two distinct genera based on 16S rRNA tree topology and sequence divergence considerations [7]. The review of these clinical cases suggests that each species is associated with a distinct clinical presentation: A. defectiva infections were seen in immunocompetent patients with endovascular infections, whereas 7 of 8 Granulicatella spp. bacteraemia occurred in immunosuppressed, mainly febrile neutropenic patients. To date, no cases of A. defectiva and only five cases of Granulicatella spp. bacteraemia in neutropenic patients have been reported. Pierard et al. described one case of $G$. adiacens bacteraemia among 62 cases of streptococcal bacteraemia in neutropenic patients [35]. In a small series published by Woo et al., three cases of $G$. adiacens bacteraemia were associated with febrile neutropenia in cancer patients [36]. Finally, one case of $G$. elegans bacteraemia was reported by Murray et al. in a febrile neutropenic cancer patient [37]. We report here the first case of "G. para-adiacens" infection in the setting of febrile neutropenia.
One and two of 8 Granulicatella spp. strains, respectively, showed reduced susceptibility and resistance to penicillin. This rate of resistance is similar to the prevalence of penicillin resistance recently described $[26,28]$. This suggests that antimicrobial susceptibility testing should be systematically done in order to select appropriate antimicrobial therapy. In severely ill patients or those with a suboptimal response to initial therapy with beta-lactam antibiotics, treatment with vancomycin should be considered. We did not observed therapeutic failures; the fatal outcome in two cases was not attributed to the infection.

All Abiotrophia and Granulicatella strains were susceptible to vancomycin. However, depending on the culture medium used for E-test method, we observed discrepant results. Overestimation of vancomycin E-test values have previously been reported for Strepococci spp., when compared to values obtained with broth or agar dilution methods [38-40]. Vancomycin E-test values should thus be interpreted with caution.

As observed in this series, Granulicatella spp. bacteraemia may occur in the setting of chemotherapy-associated mucositis and neutropenia. Oro-intestinal colonisation by Granulicatella spp. and subsequent mucositis may represent predisposing factors for bacteraemia in neutropenic patients, as it is well documented for viridans streptococci $[41,42]$. The absence of cases of bacteraemia due to $A$. defectiva in neutropenic patients could reflect a lower frequency of oral colonisation by this species in comparison to Granulicatella spp. In one study, the rates of oral colonisation in healthy students were $11.8 \%$ and $87.1 \%$ for $A$. defectiva and G. adiacens, respectively [9].

\section{Conclusion}

We report six cases of bacteraemia due to Granulicatella spp. in febrile neutropenic patients. Chemotherapyinduced neutropenia and oral mucositis may represent predisposing factors. Granulicatella spp. should be considered as a possible agent of bacteraemia in neutropenic cancer patients.

\section{Competing interests}

The author(s) declare that they have no competing interests.

\section{Authors' contributions}

LS collected the clinical data, carried out the 16S rRNA sequencing and wrote the draft of the manuscript. AW, JME and GP did the microbiological studies. KJ and GG participated in the $16 \mathrm{~S}$ rRNA sequencing. JME, GG, JB, TC and GP provided input into subsequent drafts of this manuscript. All authors read and approved the final version of manuscript. 


\section{Additional material}

\section{Additional File 1}

Clinical characteristics of 11 patients with bloodstream and endovascular infections due to Abiotrophia defectiva and Granulicatella spp. This table summarizes patients' age, sex, clinical diagnosis, species identification, Genbank accession number, underlying conditions, predisposing factors, antimicrobial therapy and outcome.

Click here for file

[http://www.biomedcentral.com/content/supplementary/14712334-6-9-S1.doc]

\section{Acknowledgements}

We thank Dr Philip E. Tarr for critical reading of the manuscript.

\section{References}

I. FRENKEL A, HIRSCH W: Spontaneous development of $\mathbf{L}$ forms of streptococci requiring secretions of other bacteria or sulphydryl compounds for normal growth. Nature |96|, 191:728-730.

2. Bouvet A, Grimont F, Grimont PAD: Streptococcus defectivus sp. nov and Streptococcus adjacens sp. nov., nutritionally variant streptococci from human clinical specimens. International Journal of Systematic Bacteriology 1989, 39:290-294.

3. Kawamura Y, Hou XG, Sultana F, Liu S, Yamamoto H, Ezaki T: Transfer of Streptococcus adjacens and Streptococcus defectivus to Abiotrophia gen. nov. as Abiotrophia adiacens comb. nov. and Abiotrophia defectiva comb. nov., respectively. Int J Syst Bacteriol 1995, 45:798-803.

4. Roggenkamp A, bele-Horn M, Trebesius KH, Tretter U, Autenrieth IB, Heesemann J: Abiotrophia elegans sp. nov., a possible pathogen in patients with culture-negative endocarditis. J Clin Microbiol 1998, 36:100-104.

5. Lawson PA, Foster G, Falsen E, Sjoden B, Collins MD: Abiotrophia balaenopterae sp. nov., isolated from the minke whale (Balaenoptera acutorostrata). Int J Syst Bacteriol 1999, 49 Pt 2:503-506.

6. Kanamoto T, Sato S, Inoue M: Genetic heterogeneities and phenotypic characteristics of strains of the genus Abiotrophia and proposal of Abiotrophia para-adiacens sp. nov. J Clin Microbiol 2000, 38:492-498.

7. Collins MD, Lawson PA: The genus Abiotrophia (Kawamura et al.) is not monophyletic: proposal of Granulicatella gen. nov., Granulicatella adiacens comb. nov., Granulicatella elegans comb. nov. and Granulicatella balaenopterae comb. nov. Int J Syst Evol Microbiol 2000, 50 Pt I:365-369.

8. Mikkelsen L, Theilade E, Poulsen K: Abiotrophia species in early dental plaque. Oral Microbiol Immunol 2000, 15:263-268.

9. Ohara-Nemoto $Y$, Tajika S, Sasaki M, Kaneko M: Identification of Abiotrophia adiacens and Abiotrophia defectiva by 165 rRNA gene PCR and restriction fragment length polymorphism analysis. J Clin Microbiol 1997, 35:2458-2463.

10. Sato S, Kanamoto T, Inoue M: Abiotrophia elegans strains comprise $8 \%$ of the nutritionally variant streptococci isolated from the human mouth. J Clin Microbiol 1999, 37:2553-2556.

II. Ruoff KL: Nutritionally variant streptococci. Clin Microbiol Rev | $991,4: 184-190$

12. Christensen JJ, Facklam RR: Granulicatella and Abiotrophia species from human clinical specimens. J Clin Microbiol 200I, 39:3520-3523.

13. Brouqui P, Raoult D: Endocarditis due to rare and fastidious bacteria. Clin Microbiol Rev 200I, 14:177-207.

14. Keay L, Harmis N, Corrigan K, Sweeney D, Willcox M: Infiltrative keratitis associated with extended wear of hydrogel lenses and Abiotrophia defectiva. Cornea 2000, 19:864-869.

15. Namdari H, Kintner K, Jackson BA, Namdari S, Hughes JL, Peairs RR Savage DJ: Abiotrophia species as a cause of endophthalmitis following cataract extraction. J Clin Microbiol 1999 37:1564-1566.
16. Michelow IC, McCracken GHJ, Luckett PM, Krisher K: Abiotrophia spp. brain abscess in a child with Down's syndrome. Pediatr Infect Dis J 2000, 19:760-763.

17. Schlegel L, Merlet C, Laroche JM, Fremaux A, Geslin P: latrogenic meningitis due to Abiotrophia defectiva after myelography. Clin Infect Dis 1999, 28:155-156.

18. Biermann C, Fries G, Jehnichen P, Bhakdi S, Husmann M: Isolation of Abiotrophia adiacens from a brain abscess which developed in a patient after neurosurgery. J Clin Microbiol 1999, 37:769-77I.

19. Zenone T, Durand DV: Brain abscesses caused by Abiotrophia defectiva: complication of immunosuppressive therapy in a patient with connective-tissue disease. Scand J Infect Dis 2004 , 36:497-499.

20. Cerceo E, Christie JD, Nachamkin I, Lautenbach E: Central nervous system infections due to Abiotrophia and Granulicatella species: an emerging challenge? Diagn Microbiol Infect Dis 2004, 48: $161-165$

21. Hepburn MJ, Fraser SL, Rennie TA, Singleton CM, Delgado BJ: Septic arthritis caused by Granulicatella adiacens: diagnosis by inoculation of synovial fluid into blood culture bottles. Rheumatol lnt 2003, 23:255-257.

22. Riede U, Graber P, Ochsner PE: Granulicatella (Abiotrophia) adiacens infection associated with a total knee arthroplasty. Scand J Infect Dis 2004, 36:76I-764.

23. Wilhelm N, Sire S, Le CA, Loubinoux J, Beljerd M, Bouvet A: First case of multiple discitis and sacroiliitis due to Abiotrophia defectiva. EurJ Clin Microbiol Infect Dis 2005, 24:76-78.

24. Heath CH, Bowen SF, McCarthy JS, Dwyer B: Vertebral osteomyelitis and discitis associated with Abiotrophia adiacens (nutritionally variant streptococcus) infection. Aust N ZJ Med 1998, 28:663.

25. Rosenthal O, Woywodt A, Kirschner P, Haller H: Vertebral osteomyelitis and endocarditis of a pacemaker lead due to Granulicatella (Abiotrophia) adiacens. Infection 2002, 30:317-319.

26. Tuohy MJ, Procop GW, Washington JA: Antimicrobial susceptibility of Abiotrophia adiacens and Abiotrophia defectiva. Diagn Microbiol Infect Dis 2000, 38: I89-191.

27. Zheng X, Freeman AF, Villafranca J, Shortridge D, Beyer J, Kabat W, Dembkowski K, Shulman ST: Antimicrobial susceptibilities of invasive pediatric Abiotrophia and Granulicatella isolates. J Clin Microbiol 2004, 42:4323-4326.

28. Liao CH, Teng LJ, Hsueh PR, Chen YC, Huang LM, Chang SC, Ho SW Nutritionally variant streptococcal infections at a University Hospital in Taiwan: disease emergence and high prevalence of beta-lactam and macrolide resistance. Clin Infect Dis 2004, 38:452-455

29. Weisburg WG, Barns SM, Pelletier DA, Lane DJ: I6S ribosomal DNA amplification for phylogenetic study. J Bacteriol I99|, 173:697-703.

30. Altschul SF, Madden TL, Schaffer AA, Zhang J, Zhang Z, Miller W, Lipman DJ: Gapped BLAST and PSI-BLAST: a new generation of protein database search programs. Nucleic Acids Res 1997 , 25:3389-3402.

3I. [http://www.ncbi.nlm.nih.gov].

32. [http://www.ddbj.nig.ac.jp].

33. Kumar S, Tamura K, Jakobsen IB, Nei M: MEGA2: molecular evolutionary genetics analysis software. Bioinformatics 200I, I7:1244-1245.

34. Institute CS: Performance standards for antimicrobial susceptibility testing: fifteenth informational supplement. Clinical and laboratory Standards Institute, Wayne, Pa 2005

35. Pierard D, de MA, Lauwers S: Antibiotic susceptibility of streptococci isolated from blood from neutropenic patients. Pathol Biol (Paris) 1994, 42:47I-474.

36. Woo PC, Fung AM, Lau SK, Chan BY, Chiu SK, Teng JL, Que TL, Yung $\mathrm{RW}$, Yuen KY: Granulicatella adiacens and Abiotrophia defectiva bacteraemia characterized by I6S rRNA gene sequencing. J Med Microbiol 2003, 52:137-I40.

37. Murray CK, Walter EA, Crawford S, McElmeel ML, Jorgensen JH: Abiotrophia bacteremia in a patient with neutropenic fever and antimicrobial susceptibility testing of Abiotrophia isolates. Clin Infect Dis 200I, 32:EI 40-EI42.

38. Smith A, Jackson MS, Kennedy H: Antimicrobial susceptibility of viridans group streptococcal blood isolates to eight antimicrobial agents. Scand J Infect Dis 2004, 36:259-263. 
39. Rosser SJ, Alfa MJ, Hoban S, Kennedy J, Harding GK: E test versus agar dilution for antimicrobial susceptibility testing of viridans group streptococci. J Clin Microbiol 1999, 37:26-30.

40. Hashemi FB, Schutze GE, Mason EOJ: Discrepancies between results by $E$-test and standard microbroth dilution testing of Streptococcus pneumoniae for susceptibility to vancomycin. J Clin Microbiol 1996, 34: I546-I547.

41. Marron A, Carratala J, Gonzalez-Barca E, Fernandez-Sevilla A, Alcaide F, Gudiol F: Serious complications of bacteremia caused by Viridans streptococci in neutropenic patients with cancer. Clin Infect Dis 2000, 3 I: I I 26- I I30.

42. Richard P, Amador DV, Moreau P, Milpied N, Felice MP, Daeschler T, Harousseau JL, Richet $\mathrm{H}$ : Viridans streptococcal bacteraemia in patients with neutropenia. Lancet 1995, 345:1607-1609.

\section{Pre-publication history}

The pre-publication history for this paper can be accessed here:

http://www.biomedcentral.com/1471-2334/6/9/prepub

Publish with Bio Med Central and every scientist can read your work free of charge

"BioMed Central will be the most significant development for disseminating the results of biomedical research in our lifetime. "

Sir Paul Nurse, Cancer Research UK

Your research papers will be:

- available free of charge to the entire biomedical community

- peer reviewed and published immediately upon acceptance

- cited in PubMed and archived on PubMed Central

- yours - you keep the copyright

Submit your manuscript here:

http://www.biomedcentral.com/info/publishing_adv.asp 